\title{
Treatment of chronic migraine with transcutaneous stimulation of the auricular branch of the vagal nerve (auricular t-VNS): a randomized, monocentric clinical trial
}

\author{
Andreas Straube ${ }^{1 *}$, J. Ellrich ${ }^{2,3}$, O. Eren ${ }^{1}$, B. Blum ${ }^{1}$ and R. Ruscheweyh ${ }^{1}$
}

\begin{abstract}
Background: Aim of the study was assessment of efficacy and safety of transcutaneous stimulation of the auricular branch of the vagal nerve (t-VNS) in the treatment of chronic migraine.

Methods: A monocentric, randomized, controlled, double-blind study was conducted. After one month of baseline, chronic migraine patients were randomized to receive $25 \mathrm{~Hz}$ or $1 \mathrm{~Hz}$ stimulation of the sensory vagal area at the left ear by a handhold battery driven stimulator for $4 \mathrm{~h} /$ day during 3 months. Headache days per 28 days were compared between baseline and the last month of treatment and the number of days with acute medication was recorded The Headache Impact Test (HIT-6) and the Migraine Disability Assessment (MIDAS) questionnaires were used to assess headache-related disability.

Results: Of 46 randomized patients, 40 finished the study (per protocol). In the per protocol analysis, patients in the $1 \mathrm{~Hz}$ group had a significantly larger reduction in headache days per 28 days than patients in the $25 \mathrm{~Hz}$ group $(-7.0 \pm 4.6$ vs. $-3.3 \pm 5.4$ days, $p=0.035) .29 .4 \%$ of the patients in the $1 \mathrm{~Hz}$ group had a $\geq 50 \%$ reduction in headache days vs. $13.3 \%$ in the $25 \mathrm{~Hz}$ group. HIT-6 and MIDAS scores were significantly improved in both groups, without group differences. There were no serious treatment-related adverse events.

Conclusion: Treatment of chronic migraine by t-VNS at $1 \mathrm{~Hz}$ was safe and effective. The mean reduction of headache days after 12 weeks of treatment exceeded that reported for other nerve stimulating procedures.
\end{abstract}

Keywords: Sensory nerve; Neuromodulation; Clinical study; Chronic headache; Electrical pulses

\section{Background}

Migraine is a frequent neurological disorder. In some patients, episodic migraine (with $<15$ headache days per month) evolves towards chronic migraine, which is characterized by $\geq 15$ headache days per month of which $\geq 8$ have migraine-like features [1], see also: http://ihs-classification.org/de/0_downloads/. Chronic migraine affects approximately 1.3 to $2.4 \%$ of the general population [2]. It is associated with significant disability and reduced health-related quality of life and often complicated by

\footnotetext{
* Correspondence: Andreas.Straube@med.uni-muenchen.de

${ }^{1}$ Klinik und Poliklinik für Neurologie, Oberbayerisches Kopfschmerzzentrum, Klinikum Großhadern, Ludwig-Maximilians-Universität München, Marchioninistr. 15, 81377 Munich, Germany

Full list of author information is available at the end of the article
}

overuse of acute pain medications [3, 4]. Up to now, randomized controlled trials showing a significant effect in the treatment specifically of chronic migraine have been published only for topiramate and onabotulinumtoxin A $[5,6]$. Treatment of chronic migraine is often difficult, with significant numbers of patients not responding to pharmacological management.

In recent years, neuromodulation was introduced in the treatment of headache [7]. Invasive occipital nerve stimulation (ONS) has been investigated for the treatment of chronic migraine, with inconsistent results [8-10]. Significant reduction in headache days was demonstrated in only one of the three studies, which however did not meet its primary endpoint (a $50 \%$ reduction of mean daily pain ratings) [10]. A major disadvantage of ONS is the safety 
profile with frequent adverse events such as infections, lead migration or lead disconnection $[8,10]$. This is also the reason why in some health markets the reimbursement of ONS was stopped by the regulatory administration. Thus, less invasive forms of neuromodulation such as transcutaneous electrical nerve stimulation are under investigation. For example, supraorbital transcutaneous stimulation for 3 months has been shown to be effective for the preventive treatment of episodic migraine (active treatment: $38 \%$ responders, sham: $12 \%$ responders, $\mathrm{p}<0.05)$ [11].

Vagal nerve stimulation using implanted electrodes is used as a treatment option in otherwise therapyrefractory epilepsy and depression [12]. Case reports and small series of patients who received an implanted vagal nerve stimulator for treatment of epilepsy and had comorbid migraine suggest that VNS may have a preventive effect in migraine [13-16]. A recently developed medical device $\left(\mathrm{NEMOS}^{\oplus}\right.$, cerbomed, Erlangen, Germany) allows for non-invasive, transcutaneous stimulation of the auricular branch of the vagus nerve (auricular t-VNS) using a special ear electrode. Auricular t-VNS excites thick myelinated sensory A $\beta$-fiber afferents in the vagal nerve, activating the nucleus of the solitary tract $[17,18]$. Effects on autonomous activity have been demonstrated in healthy subjects where auricular t-VNS increases heart rate variability [19]. Anticonvulsive effects in rodents are similar to those achieved with invasive VNS [18]. Functional imaging during auricular t-VNS has shown a pattern consistent with afferent vagal stimulation [20, 21]. Both invasive VNS and auricular $\mathrm{t}$-VNS reduce pinprick and pressure pain in humans $[22,23]$. In addition, a recent observational study has suggested that $\mathrm{t}-\mathrm{VNS}$ to the right cervical branch of the vagus nerve (cervical t-VNS) may be effective for acute migraine treatment [24]. In the present study, we investigated the effect of auricular t-VNS on chronic migraine.

\section{Methods}

This was a monocentric, prospective, double-blind, randomized, parallel-group, controlled trial analyzed both on intention-to-treat basis (ITT), and on per protocol basis (PP). The trial was conducted in a German tertiary headache outpatient clinic (Department of Neurology, University of Munich). The study was approved by the ethics committee of the medical faculty of the University of Munich and written informed consent was obtained from all participants. The study is registered in the German Clinical Trials Register (DRKS00003681).

\section{Study participants}

Men or women between 18 and 70 years with a diagnosis of chronic migraine according to the ICHD-IIR (code A1.5.1.) (http://ihs-classification.org/de/0_downloads/), duration of $\geq 6$ months, no migraine-prophylactic medication or stable migraine-prophylactic medication for $\geq 1$ month, and stable acute medication were eligible, medication overuse was not an exclusion criterion.

Patients were excluded if they suffered from other primary or secondary headaches, severe neurologic or psychiatric disorders including opioid- or tranquilizer-dependency, cranio-mandibulary dysfunction, fibromyalgia, had a Beck's Depression Inventory (BDI [25]) score $>25$ at the screening visit, anatomic or pathologic changes at the left outer ear, currently participated in another clinical trial, or were unable to keep a headache diary. Pregnant or breast-feeding women were also excluded. A pregnancy test was performed at the screening visit in women of childbearing potential and they were required to use a reliable means of contraception. In addition, patients who had less than 15 headache days per 28 days during the 4-week baseline period were excluded.

\section{Study design (Fig. 1)}

The study consisted of a 4-week screening period ("baseline") followed by a 12-week randomized, doubleblind, parallel-group treatment period with either $1 \mathrm{~Hz}$ or $25 \mathrm{~Hz}$ tVNS with the NEMOS ${ }^{\bullet}$ device (Fig. 2). Adverse events were recorded at visits 2 to 6 . Compliance with stimulation was checked at visits 3 to 6 by reading out the $\mathrm{NEMOS}^{\bullet}$ device and quantified in percent of the

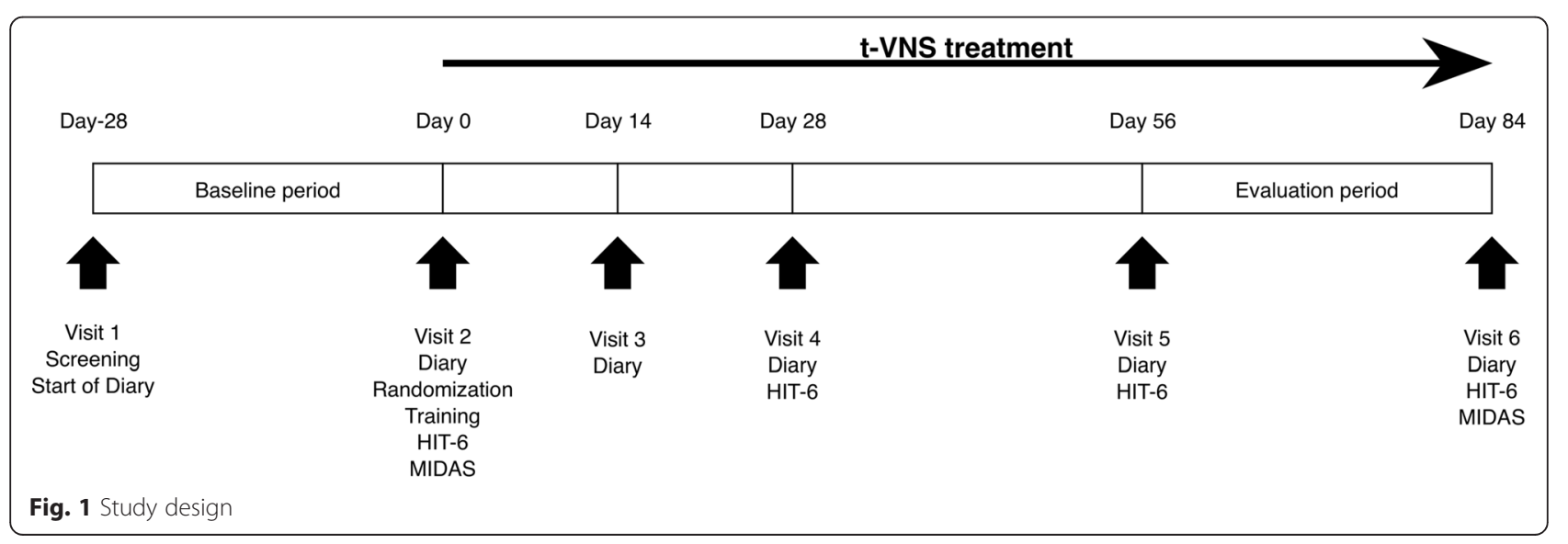




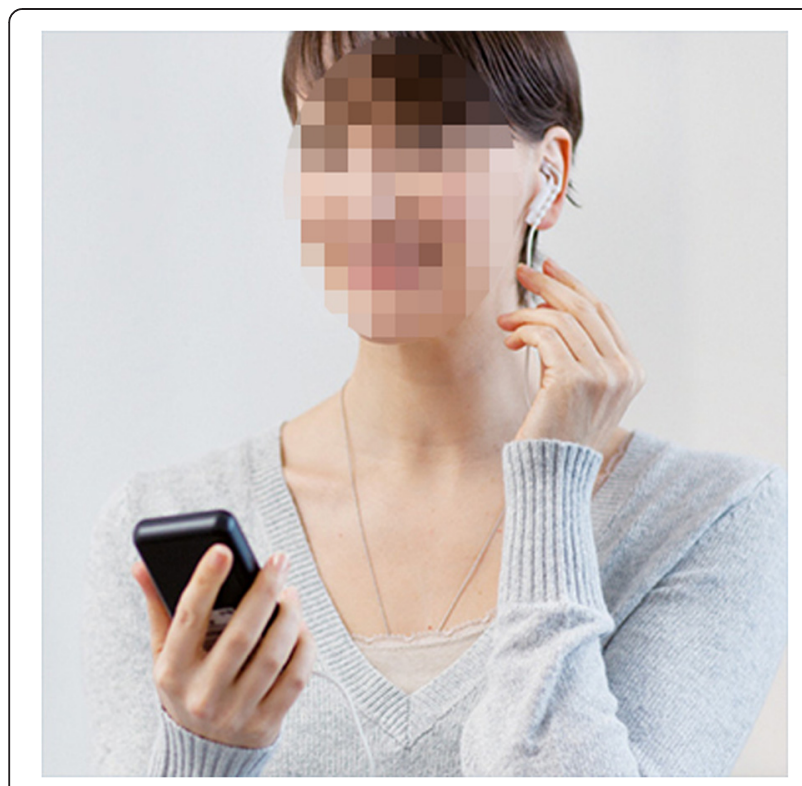

Fig. 2 NEMOS ${ }^{\circledR}$ device and positioning of the electrode for stimulation of the vagus afferents at the concha

intended daily stimulation time $(4 \mathrm{~h})$. Re-training was administered during visits 3 to 6 as necessary. The Migraine Disability Assessment (MIDAS [26]) and the Headache Impact Test (HIT-6 [27]) were filled in by the patient as indicated in Fig. 1. Patients kept a paper-and-pencil headache diary during the entire period, handing in their diaries and receiving a fresh sheet at each visit. In the diary, patients indicated for every day (1) headache duration in hours, (2) headache intensity (on a 0 to 10 numerical rating scale: 0 , no pain; 10 , strongest pain imaginable), and (3) intake of acute headache medication (analgesics, triptans).

Sample size calculations were based on published studies on successful pharmacological treatment of chronic migraine (mean effect size: $-4,68$ headache days/month after removal of the placebo effect) $[5,6,28,29]$. To detect an effect of this size with an $\alpha$ error of 0.05 and a power of 0.80 , a group size of 49 patients per treatment group was estimated, including $10 \%$ drop-out. An interims analysis after 46 patients was planned. Since patient recruitment was slower than expected, the sponsor decided to terminate the study at the interims analysis, and no further patients were enrolled.

\section{Neurostimulation}

The NEMOS ${ }^{\circ}$-VNS device (Cerbomed, Erlangen, Germany) is a transcutaneous vagus nerve stimulator designed for electrical stimulation at the concha of the outer ear, which receives sensory innervation from the auricular branch of the vagal nerve (Fig. 2). The NEMOS ${ }^{\circ}$ device has received the CE mark for treatment of pain (CE0408) and is registered in the European Databank on Medical Devices (EUDAMED, CIV-11-09-002381). It consists of a handheld, battery driven electrical stimulator connected to an ear electrode placed in contact with the skin of the concha. Impedance is measured automatically and insufficient electrode contact with the skin evokes an alarm. During stimulation, series of electrical pulses (pulse width: $250 \mu \mathrm{s}$, frequency: $1 \mathrm{~Hz}$ or $25 \mathrm{~Hz}$, duty cycle: $30 \mathrm{~s}$ on, $30 \mathrm{~s}$ off, to avoid habituation) are applied to the skin of the concha. Stimulus intensity was individually fitted during visit 2 to elicit a tingling but not painful sensation, and could later be adjusted by the patient as needed. Patients were asked to stimulate for a total of $4 \mathrm{~h}$ per day (in sessions of 1 to $4 \mathrm{~h}$, a specific distribution over the day or interval between sessions was not required), and were free to stimulate for an additional hour if they thought this was useful, e.g. for treatment of acute headache. The effect of such acute treatment was not recorded. Stimulation parameters of the $25 \mathrm{~Hz}$ group were chosen so that with $4 \mathrm{~h}$ of daily stimulation, the number of electrical stimuli per day would be similar to those normally used for invasive vagal nerve stimulation in patients with epilepsy. The $1 \mathrm{~Hz}$ stimulation was intended as an active control. The active control was chosen in order to avoid un-blinding of the subjects.

\section{Primary and secondary outcome parameters}

All outcome measures refer to change from baseline (the 4-week period between visits 1 and 2) to the evaluation period (the 4-week period between visits 5 and 6, Fig. 1). The primary outcome measure was mean change in headache days per 28 days. A headache day was defined as a calendar day with headache of $\geq 4$ h duration or headache successfully aborted by acute headache medication or any other treatment known to be typically effective in the specific patient (e.g. sleep, progressive relaxation exercises).

Secondary outcome parameters were: (1) percentage of "responders" (subjects having at least $50 \%$ reduction of headache days per 28 days from baseline to evaluation); (2) change in mean headache intensity on days with headache; (3) change in days with acute headache medication intake per 28 days; (4) change in headache-related disability, as assessed by the MIDAS and HIT-6 questionnaires; (5) number and type of adverse events.

\section{Statistical analysis}

Mean \pm standard deviation (SD) is reported unless stated otherwise. The threshold for significance of statistical comparisons was set at $\mathrm{p}<0.05$. Statistical analysis was performed both on ITT and on per protocol basis (PP). For the ITT analysis, a last observation carried forward approach was used for patients who dropped out during the course of the study.

Group comparisons at baseline, of duration of the treatment period, compliance or number of patients affected by adverse events were done using Mann-Whitney $U$-Test or Fisher's exact test as appropriate. Analysis of the primary endpoint was done using an analysis of covariance (ANCOVA) model with the factors treatment group $(1 \mathrm{~Hz}$ vs. $25 \mathrm{~Hz}$ ) and sex as categorical variables and baseline 
values as covariate. The same type of ANCOVA was used for the analysis of the following secondary outcome parameters: change in mean headache intensity, change in days with acute headache medication intake per 28 days and change in MIDAS and HIT-6 scores. The number of responders was compared between groups using a logistic regression model that included treatment group and sex as factor and the number of headache days per 28 days at baseline as covariate. An estimate of the treatment odds ratio (Wald method) was derived from this model.

\section{Results}

The study was conducted between March 2012 and July 2014. A total of 46 patients were randomized to the $1 \mathrm{~Hz}$ group $(n=22)$ or the $25 \mathrm{~Hz}$ group $(n=24$, ITT). 6 patients dropped out during the study. Reasons for dropouts were: adverse events in 4 patients (treatment-related stimulation site ulcer in 3 patients, gastrectomy not related to treatment in 1 patient), insufficient compliance in 1 patient, patient's request in 1 patient. One additional patient was excluded from the per protocol (PP) analysis after the end of the study because of violation of inclusion criteria $(<15$ headache days per 28 days in the screening period). This left 17 patients in the $1 \mathrm{~Hz}$ group and 22 patients in the $25 \mathrm{~Hz}$ group for the PP analysis (Fig. 3) Demographic and headache characteristics of the population are shown in Table 1. There were no significant differences between both groups.

\section{Primary outcome measure}

PP-analysis indicated a significant decrease in headache days per 28 days from baseline to evaluation, which was significantly larger in the $1 \mathrm{~Hz}$ group than in the $25 \mathrm{~Hz}$ group $(\mathrm{F}[35]=4.82, p=0.035$, Table 2$)$. In the $1 \mathrm{~Hz}$ group, the reduction amounted to -7.0 days per 28 days (36.4\% reduction from baseline), while the $25 \mathrm{~Hz}$ group reached only -3.3 days (17.4 \% reduction from baseline). In the ITT analysis, there also was a significant decrease in headache days per 28 days in both groups, but no significant group difference $(\mathrm{F}[42]=$ $2.94, p=0.094$, Table 2). Visual inspection of headache days per 28 days over the treatment period revealed a continuous decrease in the $1 \mathrm{~Hz}$ group, while a steady state was reached after 14 days in the $25 \mathrm{~Hz}$ group (Fig. 4).

\section{Secondary outcome measures}

Results of secondary outcome measures and the corresponding statistics are summarized in Table 2. The number of responders ( $>50 \%$ improvement in headache days) was in the $1 \mathrm{~Hz}$ group (PP) $29.4 \%$ and in the $25 \mathrm{~Hz}$ group (PP) $13.6 \%$. Headache intensity was not significantly changed by t-VNS in either treatment group, and there were no group differences. The number of days with intake of acute headache medication as well as the MIDAS and HIT-6 scores were significantly reduced in both treatment groups, there were no group differences.

\section{Treatment duration and compliance}

Results and statistics are listed in Table 3. Duration of the treatment period was similar between groups. The average number of stimulated hours per day during the treatment period was around 3.4 in all groups, corresponding to around $85 \%$ of the requested 4 h of daily

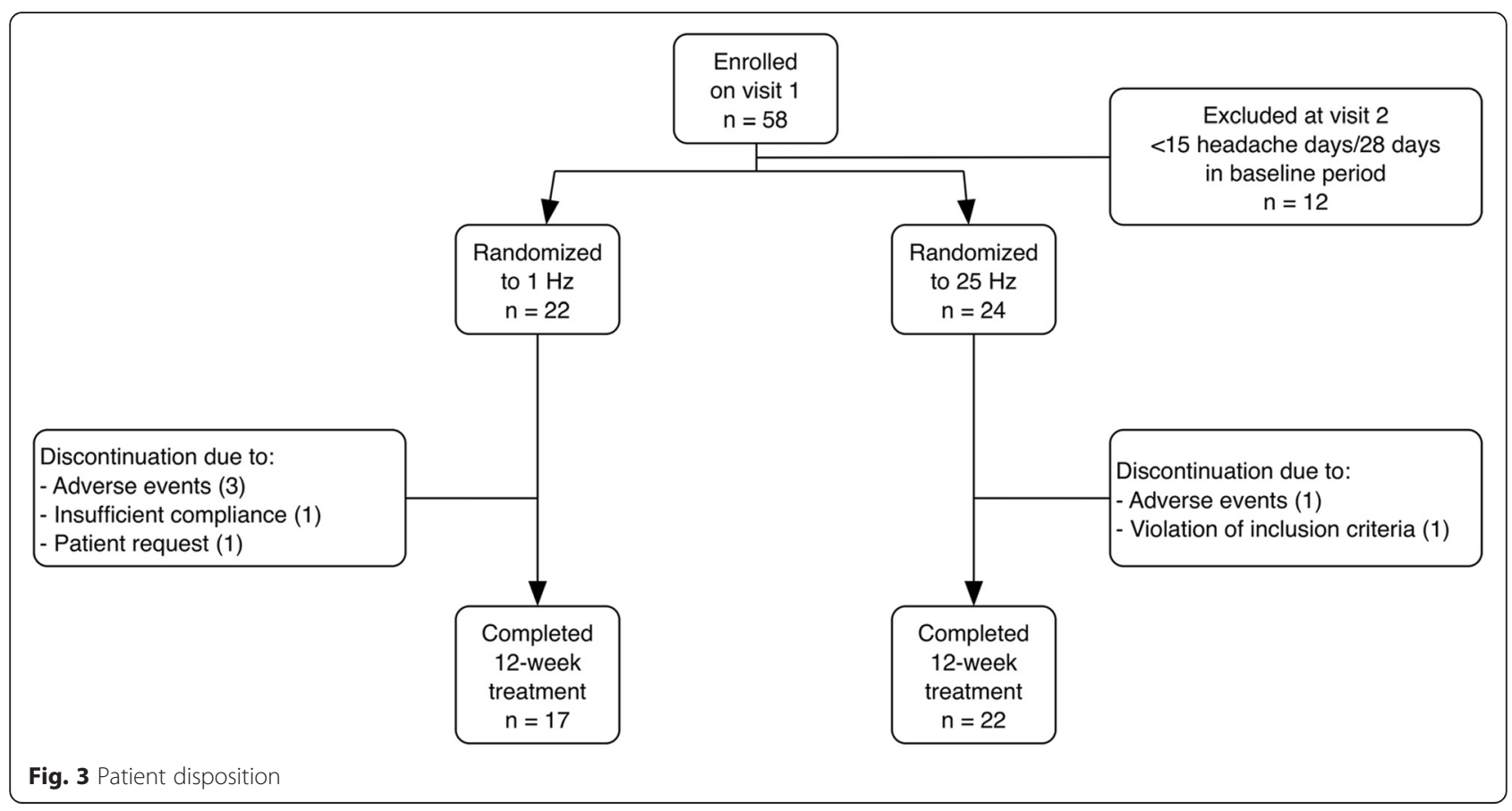


Table 1 Baseline characteristics of the cohort

\begin{tabular}{|c|c|c|c|c|c|c|}
\hline & \multicolumn{3}{|c|}{ Intention-to-treat analysis } & \multicolumn{3}{|c|}{ Per protocol analysis } \\
\hline & $1 \mathrm{~Hz}(n=22)$ & $25 \mathrm{~Hz}(n=24)$ & Group comparison & $1 \mathrm{~Hz}(n=17)$ & $25 \mathrm{~Hz}(n=22)$ & Group comparison \\
\hline Age & $43.8 \pm 11.5$ & $39.3 \pm 12.4$ & $p=0.21$ & $44.1 \pm 11.4$ & $39.0 \pm 12.5$ & $p=0.21$ \\
\hline Females & 18 & 21 & $p=0.69$ & 13 & 19 & $p=0.68$ \\
\hline Headache days/28 days & $19.4 \pm 4.0$ & $18.9 \pm 5.1$ & $p=0.47$ & $19.1 \pm 3.7$ & $19.2 \pm 4.7$ & $p=0.66$ \\
\hline Headache intensity (NRS: 0-10) & $5.2 \pm 1.5$ & $5.0 \pm 1.5$ & $p=0.73$ & $5.0 \pm 1.5$ & $5.0 \pm 1.5$ & $p=0.98$ \\
\hline Migraine history (years) & $27.1 \pm 13.0$ & $20.4 \pm 12.1$ & $p=0.08$ & $27.8 \pm 11.5$ & $21.4 \pm 12.1$ & $p=0.11$ \\
\hline Days with acute headache medication/28 days & $10.3 \pm 6.4$ & $8.2 \pm 4.9$ & $p=0.24$ & $11.1 \pm 6.6$ & $8.6 \pm 4.8$ & $p=0.17$ \\
\hline MIDAS score & $76.8 \pm 64.8$ & $83.6 \pm 56.0$ & $p=0.55$ & $77.2 \pm 70.1$ & $82.1 \pm 58.0$ & $p=0.71$ \\
\hline HIT-6 score & $64.3 \pm 4.7$ & $66.0 \pm 4.1$ & $p=0.25$ & $64.8 \pm 5.0$ & $66.0 \pm 4.2$ & $p=0.55$ \\
\hline BDI & $6.9 \pm 5.7$ & $7.9 \pm 5.6$ & $p=0.59$ & $6.9 \pm 5.9$ & $7.2 \pm 5.0$ & $p=0.95$ \\
\hline
\end{tabular}

Demographic and headache characteristics assessed at the first visit or during the baseline period (4 weeks) are given. Values are mean \pm SD or numbers of subjects. Results of Mann-Whitney $U$ test or Fisher's Exact test are given. Headache intensity (NRS: numerical rating scale 0-10) MIDAS migraine disability assessment, HIT headache impact test, BDI beck's depression inventory

stimulation, indicating good compliance with treatment. There were no significant group differences.

\section{Safety and tolerability}

Adverse events (AEs) were analysed in the full analysis set (safety set) and summarized in Table 4. The number of treatment emergent AEs (AEs occurring after initiation of treatment) was higher in the $25 \mathrm{~Hz}$ group (112 events, 76 treatment-related events) as compared to the $1 \mathrm{~Hz}$ group (67 events, 39 treatment-related events, Table 4),. Most AEs were mild or moderate in severity and resolved without sequelae. The most frequent treatment-related $\mathrm{AE}$ were local problems at the stimulation site, such as mild or moderate pain, paresthesia, or pruritus during or after stimulation, and erythema, ulcer or scab (31 events in 10 patients in the $1 \mathrm{~Hz}$ group, 70 events in 17 patients in the
$25 \mathrm{~Hz}$ group, $p=0.14)$. Treatment-related AEs leading to discontinuation of the study were stimulation site ulcer (accompanied by pain, paresthesia, or pruritus) in 2 patients of the $1 \mathrm{~Hz}$ group and in 1 patient of the $25 \mathrm{~Hz}$ group. These three cases of application site ulcer occurred early during the study. After that, patients were asked to specially care for the skin of their ear after each use of the NEMOS ${ }^{\circ}$ device, using a custom rich skin cream, and no more cases of application site ulcer occurred. There were no treatmentrelated SAEs. Three SAEs, leading to hospitalization of the patient, were recorded during the whole study (infectious mononucleosis, gastrectomy, intervertebral disc protrusion).

\section{Discussion}

The present monocentric, randomized, controlled, doubleblind, parallel-group clinical trial provides evidence that

Table 2 Results of primary and secondary treatment outcome measures

\begin{tabular}{|c|c|c|c|c|c|c|}
\hline & \multicolumn{3}{|c|}{ Intention-t-treat analysis } & \multicolumn{3}{|c|}{$\underline{\text { Per protocol analysis }}$} \\
\hline & $1 \mathrm{~Hz}(n=22)$ & $25 \mathrm{~Hz}(n=24)$ & Group comparison & $1 \mathrm{~Hz}(n=17)$ & $25 \mathrm{~Hz}(n=22)$ & Group comparison \\
\hline \multirow[t]{2}{*}{ Change in headache days/28 days } & $-5.6 \pm 5.0$ & $-3.0 \pm 5.3$ & $F[42]=2.94$ & $-7.0 \pm 4.6$ & $-3.3 \pm 5.4$ & $\mathrm{~F}[35]=4.82$ \\
\hline & $(-5.9 ;-0.5)$ & $(-8.5 ;-3.2)$ & $p=0.094$ & $(-9.6 ;-4.1)$ & $(-5.9 ;-0.4)$ & $p=0.035$ \\
\hline \multirow{2}{*}{$\begin{array}{l}\text { Responder ( } 50 \% \text { reduction in } \\
\text { headache days) }\end{array}$} & \multirow[t]{2}{*}{$5(22.7 \%)$} & \multirow[t]{2}{*}{$3(12.5 \%)$} & $O R=2.44$ & \multirow[t]{2}{*}{$5(29.4 \%)$} & \multirow[t]{2}{*}{$3(13.6 \%)$} & $O R=3.21$ \\
\hline & & & $p=0.29$ & & & $p=0.18$ \\
\hline \multirow{2}{*}{$\begin{array}{l}\text { Change in headache intensity } \\
\text { (NRS } 0-10 \text { ) }\end{array}$} & $-0.1 \pm 1.1(n=20)$ & $0.2 \pm 1.0$ & $F[40]=0.30$ & $0.02 \pm 1.2(n=15)$ & $0.2 \pm 1.0$ & $F[33]=0.28$ \\
\hline & $(-0.2 ; 0.9)$ & $(-0.4 ; 0.7)$ & $p=0.58$ & $(-0.4 ; 0.8)$ & $(-0.2 ; 0.9)$ & $p=0.60$ \\
\hline \multirow{2}{*}{$\begin{array}{l}\text { Change in days with acute headache } \\
\text { medication in } 28 \text { days }\end{array}$} & $-2.0 \pm 4.2$ & $-1.3 \pm 4.4$ & $F[42]=0.01$ & $-2.7 \pm 4.5$ & $-1.6 \pm 4.1$ & $F[35]<0.01$ \\
\hline & $(-4.2 ;-0.3)$ & $(-4.4 ;-0.3)$ & $p=0.91$ & $(-4.7 ;-0.4)$ & $(-4.7 ;-0.3)$ & $p=0.96$ \\
\hline \multirow[t]{2}{*}{ Change in MIDAS score } & $-18.7 \pm 28.0$ & $-21.8 \pm 54.5$ & $F[42]<0.01$ & $-24.2 \pm 29.8$ & $-26.5 \pm 53.9$ & $F[35]<0.01$ \\
\hline & $(-38.6 ;-0.9)$ & $(-39.2 ;-0.8)$ & $p=0.98$ & $(-43.2 ;-4.0)$ & $(-42.1 ;-3.7)$ & $p=0.96$ \\
\hline \multirow[t]{2}{*}{ Change in HIT-6 score } & $-2.5 \pm 6.8$ & $-3.8 \pm 5.5$ & $F[42]=0.12$ & $-3.8 \pm 7.1$ & $-3.9 \pm 5.69$ & $F[35]=0.01$ \\
\hline & $(-6.7 ;-0.7)$ & $(-7.3 ;-1.2)$ & $p=0.73$ & $(-7.8 ;-1.1)$ & $(-7.6 ;-1.0)$ & $p=0.93$ \\
\hline
\end{tabular}

Change refers to change from the 4-week baseline period to the last 4 weeks of the 12 -week treatment period. Means, SDs and $95 \%$ confidence intervals are given. For the responder analysis, numbers of subjects and percent of the total group are given. Significant differences are marked in bold. Number of subjects is given in parentheses, where different from the total group. Primary outcome parameter: change in headache days/28 days

MIDAS migraine disability assessment, HIT headache impact test, NRS numerical rating scale 0-10 


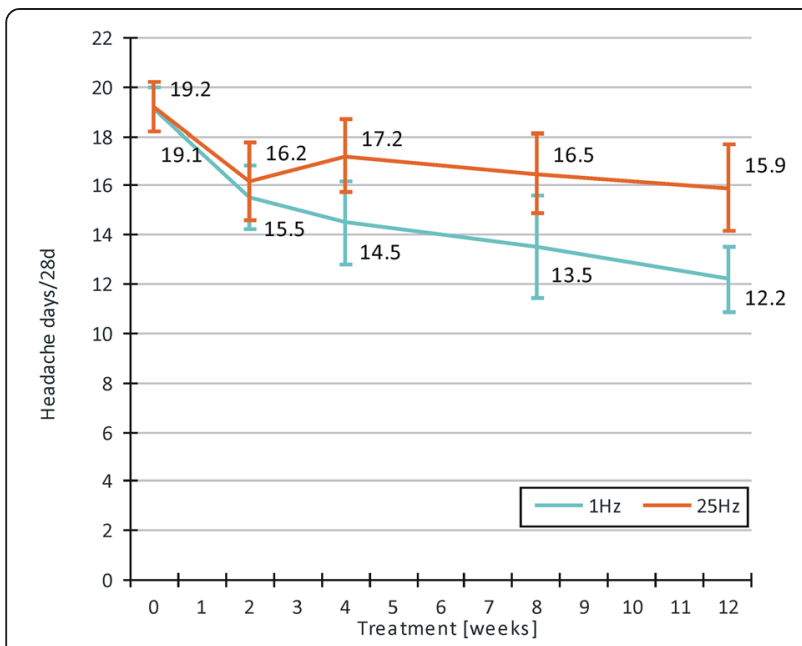

Fig. 4 Mean course of number of headache days per 28 days during t-VNS treatment. Results of the per protocol set are shown $(1 \mathrm{~Hz}$ : $n=17,25 \mathrm{~Hz}: n=22)$. Values are mean \pm SEM. Mean values are also given in the figure

daily treatment with auricular t-VNS is effective in chronic migraine.

Both in the $1 \mathrm{~Hz}$ and the $25 \mathrm{~Hz}$ group the number of headache days per 28 days decreased significantly by 7.0 and 3.3 days, respectively (PP-analysis, Table 2), with a significantly larger reduction in the $1 \mathrm{~Hz}$ compared to the $25 \mathrm{~Hz}$ group $(p=0.035) .29 .4 \%$ of the patients in the $1 \mathrm{~Hz}$ group and $13.6 \%$ of the patients in the $25 \mathrm{~Hz}$ group achieved a reduction of more than $50 \%$ in headache days ("responder"). With an absolute reduction in headache days per 28 days by 7.0 in the $1 \mathrm{~Hz}$ group and a mean group difference of 2.7 headache days, the effect of auricular t-VNS was comparable to the effects of topiramate and onabotulinumtoxin A versus placebo. Previous trials in chronic migraine with topiramate for 4 months have shown a reduction in headache days per month of 3.5 and 6.4 days in the verum group, which exceeded the effect in the placebo group by 3.7 and 1.7 days, respectively [6, 30]. In the large PREEMPT trials onabotulinumtoxin A was able to reduce the number of headache days per month in chronic migraine patients by 9.0 and 7.8 days after 6 months, which exceeded the placebo effect by 2.3 and 1.4 days, respectively $[5,31]$. Compared to previous trials investigating neurostimulation devices the results are favorable. In the ONS trials for chronic migraine, reduction of headache days after 3 months was by 6.7, 5.5 and 6.1 days in the verum group, which exceeded the sham group by 5.2, 1.6 and 3.1 days, respectively [8-10]. However, none of these studies reached significance for its primary end point. Transcutaneous supraorbital neurostimulation has so far only been tested in episodic migraine, achieving a reduction by 2.5 headache days from a baseline of 7.8 headache days, which was 2.3 days more than placebo [11].

It has to be mentioned that the study was planned as a trial with an active comparator in order to be sure that the patients were blinded and that we expected that the $25 \mathrm{~Hz}$ stimulation would be more effective than the $1 \mathrm{~Hz}$ stimulation, corresponding to the results from the use of invasive VNS in epilepsy [32, 33]. This means that it is very unlikely that partial unblinding may have affected the results, as the local sensation is more intense with $25 \mathrm{~Hz}$ stimulation, and the study physicians expected the $25 \mathrm{~Hz}$ stimulation to be more effective. However, it is not clear why the $1 \mathrm{~Hz}$ stimulation was more effective than the $25 \mathrm{~Hz}$ stimulation. The mechanisms by which VNS influences chronic migraine may be different from those in epilepsy. In addition, activation of central nervous system structures by stimulation of thickly myelinated sensory fibers in the auricular branch of the vagus nerve may require different stimulation patterns than the cervical branch, which is a mixed nerve with myelinated and non-myelinated efferent as well as afferent fibers. As no dose-response or frequency-response data are available for any neurostimulation method in migraine treatment, the question whether frequency or total number of stimuli influence the result remains open.

Analgesic effects of electrical low-frequency stimulation (LFS) in various pain models have been demonstrated in man and rodents [34]. Electrical pulse series with optimum frequency of $1 \mathrm{~Hz}$ for 20 min significantly suppressed nociceptive signaling and pain perception by approximately $40 \%$ for hours [35, 36]. This phenomenon of long-term depression (LTD) has been shown in the spinal system [37-41] and in the craniofacial area [42-44]. Stimulation parameter of $\mathrm{t}-\mathrm{VNS}$ in the present study resemble electrical LFS and could have provoked LTD of nociceptive processing in the spinal trigeminal nucleus that plays a critical role in migraine pain [45]. Actually, the auriculotemporal nerve, a branch of the trigeminal nerve, supplies the outer ear and could, therefore, mediate access of electrically evoked neural

Table 3 Duration of treatment period and compliance with stimulation during the treatment period

\begin{tabular}{|c|c|c|c|c|c|c|}
\hline & \multicolumn{3}{|c|}{ Intention-to-treat analysis } & \multicolumn{3}{|c|}{ Per protocol analysis } \\
\hline & $1 \mathrm{~Hz}(n=22)$ & $25 \mathrm{~Hz}(n=24)$ & Group comparison & $1 \mathrm{~Hz}(n=17)$ & $25 \mathrm{~Hz}(n=23)$ & Group comparison \\
\hline Treatment period (days) & $77.9 \pm 25.8$ & $85.7 \pm 11.4$ & $p=0.22$ & $89.0 \pm 8.4$ & $87.5 \pm 7.5$ & $p=0.67$ \\
\hline Average number of stimulated hours per day & $3.42 \pm 0.59$ & $3.44 \pm 0.61$ & $p=0.69$ & $3.34 \pm 0.62$ & $3.44 \pm 0.62$ & $p=0.51$ \\
\hline
\end{tabular}

Mean \pm SD values are given. Treatment period indicates the number of days between visits 2 and 6 . The average number of stimulated hours per day of the treatment period is given. Patients were requested to stimulate $4 \mathrm{~h}$ per day during the treatment period. The real average stimulation time per day was slightly lower 
Table 4 Overview of adverse events (safety set)

\begin{tabular}{|c|c|c|c|c|}
\hline & \multicolumn{2}{|l|}{$1 \mathrm{~Hz}(n=22)$} & \multicolumn{2}{|l|}{$25 \mathrm{~Hz}(n=24)$} \\
\hline & Number of events & Number of patients (\%) & Number of events & Number of patients (\%) \\
\hline Treatment emergent AEs & 67 & $17(77.3 \%)$ & 112 & $19(79.2 \%)$ \\
\hline Treatment-related AEs & 39 & $11(50.0 \%)$ & 76 & $17(70.8 \%)$ \\
\hline Stimulation site treatment-related TEAEs & 31 & $10(45.5 \%)$ & 70 & $17(70.8 \%)$ \\
\hline All serious AEs (including pre-treatment SAEs) & 2 & $2(9.1 \%)$ & 0 & 0 \\
\hline Serious treatment emergent AEs & 2 & $2(9.1 \%)$ & 0 & 0 \\
\hline Serious treatment-related AEs & 0 & 0 & 0 & 0 \\
\hline Treatment-related AEs leading to discontinuation of study & 8 & $4(18.2 \%)$ & 4 & 1 (4.2\%) \\
\hline Death & 0 & 0 & 0 & 0 \\
\hline
\end{tabular}

signals to brainstem nuclei of the trigeminal nerve [46]. Thus, LTD could be a mechanism that might, at least, contribute to the analgesic effect of t-VNS in the present study.

In fact, other stimulation parameters might be even more effective than the $1 \mathrm{~Hz}$ stimulation, and the $25 \mathrm{~Hz}$ stimulation might have been partially active in the present study, possibly reducing the effect in the group comparison. Indeed, $25-30 \mathrm{~Hz}$ stimulation has been shown to significantly reduce experimental pain in humans [23] and seizures in rodents [18]. In addition, in the present study both groups significantly improved in headache-related disability measures (MIDAS and HIT-6), and reduced their intake of acute headache medication, although it cannot be determined if this is due to the placebo effect or due to stimulation effects in both groups. The missing significant difference in the reduction of the MIDAS and HIT6 between the $1 \mathrm{~Hz}$ and the $25 \mathrm{~Hz}$ group is probably due to the too small sensitivity of these tests in detecting differences in quality of life. Furthermore, it is unclear if $25 \mathrm{~Hz}$ stimulation also have a mood stabilizing effect which influences the ratings in the used tests.

Furthermore, it is still not clear how vagus nerve stimulation interferes with migraine generation. One possibility is a direct or indirect inhibition of nociceptive trigeminal neurons by vagal activation. Indeed, animal data show that afferent vagal stimulation can reduce the activation of nociceptive neurons in the caudal trigeminal nucleus in response to noxious stimulation of the face or dura [47-49]. This might be due to the existence of dense reciprocal connections between the spinal trigeminal nucleus and the nucleus tractus solitarii (NTS) which is the major target of vagal afferents [50]. Responses of spinal trigeminal neurons might also be reduced by activation of the descending pain inhibitory systems. Although this has not been shown directly for the trigeminal area, animal studies showed that vagal nerve stimulation can activate descending pain inhibitory systems, probably involving projections from the NTS to the nucleus raphe magnus and the locus coeruleus, which are at the origin of serotonergic and noradrenergic descending pain inhibitory pathways [51]. Alternatively, VNS might exert migraine prophylactic actions by modifying cortical excitability. Altered cortical excitability in chronic migraine has been demonstrated in various electrophysiological measurements is thought to contribute to its pathogenesis [52]. Several lines of evidence indicate that the cortical excitability is increased in chronic migraine patients: 1 ) There is a reduced habituation of the blink reflex interictally [53]. 2) The magnetic suppression of perceptual accuracy was decreased in patients with chronic migraine compared to episodic migraine and controls which may indicate also a higher cortical excitability [54]. 3) Analysis of the high frequency somatosensory evoked potentials showed early response sensitization and late habituation, most probably due an increased coupling between thalamus and cortex in chronic migraine [55]. Afferent vagal information is relayed via the NTS and the parabrachial nucleus to several subcortical and cortical regions, including thalamus, insula and lateral prefrontal cortex. In addition, the NTS has strong projections to the locus coeruleus and the nucleus raphe magnus which provide widespread noradrenergic and serotonergic innervation of the cortex [56]. Modulation of cortical excitability via these pathways is thought to be important for the anticonvulsant effects of VNS [33]. Increased GABA levels have been found in the cerebrospinal fluid of epilepsy patients treated with VNS, suggesting an increase in inhibitory neurotransmission [57]. Auricular $\mathrm{t}$-VNS increases parasympathetic activity and/or reduces sympathetic activity [19], which might also affect cortical excitability, maybe by mechanisms similar to those assumed for the migraine preventive effects of beta-blocking agents [58]. In summary, VNS is well positioned to alter cortical excitability, especially to reduce cortical hyperexcitability. Direct evidence that this interferes with pain processing or migraine generation is currently lacking. It would be interesting to repeat the above described experiments which showed an increased cortical excitability in chronic migraine under t-VNS stimulation. A third possibility is that the antimigraine action of VNS relies on modification of transmitter release from efferent parasympathetic fibers innervating 
dural vessels, e.g. fibers stemming from the spheno-palatine ganglion. The release of neurotransmitters, especially calcitonin-gene related peptide (CGRP), at dural vessels with subsequent neurogenic inflammation and sensitization of primary afferents is thought to play an important role in migraine pathophysiology [59]. Parasympathetic fibres innervating the dura mater release vasoactive intestinal polypeptide (VIP) and pituitary adenylate cyclase-activating polypeptide (PACAP), which are potent vasodilatators and thought to contribute to sensitization of nociceptive trigeminal primary afferents. Increased peripheral blood VIP levels have been detected in chronic migraine [60], and intravenous administration of PACAP has been shown to induce migrainous headache in migraine patients [61], suggesting that both transmitters are related to migraine pathophysiology. Although auricular t-VNS stimulates only vagal afferents, there are close connections between afferent and efferent parasympathetic brainstem centers, making an influence of VNS on dural efferents likely.

A major practical advantage of auricular t-VNS is good tolerability and safety. For comparison, in the pooled topiramate trial analysis, 1 out of 4 patients ( $25 \%$ ) dropped out because of intolerable adverse effects [62]. In our study only 3 of 46 patients (7\%) dropped out due to side effects of t-VNS. All three cases occurred early in the study and were due to stimulation site ulcer which later in the study could be prevented by appropriate skin care. Another advantage of $\mathrm{t}$-VNS therapy is that it can be combined with any other drug treatment without risking cumulative adverse effects or pharmacodynamic interactions. In addition, auricular t-VNS allows patients to continue routine activities, leading to a high compliance with stimulation times (around $85 \%$ on average). However, long-term effects and sustainability of efficacy of t-VNS are still unknown and need to be demonstrated in appropriate open-label trials.

\section{Conclusions}

In conclusion, the present parallel-group randomized controlled trial, provides evidence that auricular t-VNS at $1 \mathrm{~Hz}$ for $4 \mathrm{~h}$ daily is effective for chronic migraine prevention over 3 months. The absolute reduction in headache days (7.0) and the difference between groups (2.7 headache days) is comparable to the effects of topiramate and onabotulinum toxin $\mathrm{A}$ in chronic migraine prevention. The $\mathrm{t}-\mathrm{VNS}$ treatment also results in a meaningful improvement in the quality of life as assessed by MIDAS and HIT 6. The safety profile was favourable and compliance with daily stimulation was high.

\section{Competing interests}

Cerbomed funded the study.

A. Straube has received honoraries by Pharm Allergan, Boehringer Ingelheim, Hormosan, electroCore, CerboMed. Grants from the German Science foundation, German Minister of Research and Education and the Kröner-Fresenius foundation.
J. Ellrich was employed as Chief Medical Officer by the company cerbomed $\mathrm{GmbH}$.

O. Eren has nothing to disclose

B. Blum has nothing to disclose

R. Ruscheweyh has received honaries by Pharm Allergan, MSD,

Mundipharma, Pfizer and grants from the Else Kröner Fresenius Stiftung.

\section{Authors' contributions}

The study was planned by JE and AS. AS, RR, OE, BB recruited the patients and collected the data. Statistical analysis was performed by Metronomia Clinical Research GmbH (Munich, Germany). Cerbomed supported the preparation of the figures and the layout. The paper was written by the authors and all authors participated in the decision to publish the paper and had full access to all study data. All authors read and approved the final manuscript.

\section{Acknowledgement}

The authors thank Nadine Wolf, PhD for her contribution to the clinical investigation plan and A. Hartlep, PhD and V. Koepke for their help in the preparation of the manuscript.

\section{Author details}

${ }^{1}$ Klinik und Poliklinik für Neurologie, Oberbayerisches Kopfschmerzzentrum, Klinikum Großhadern, Ludwig-Maximilians-Universität München, Marchioninistr. 15, 81377 Munich, Germany. ${ }^{2}$ Department of Health Science and Technology,

Professor Dr. med. Jens Ellrich, Aalborg University, Fredrik Bajers Vej 7D2,

DK-9220 Aalborg, Denmark. ${ }^{3}$ Cerbomed GmbH, Medical Valley Center, Henkestr. 91, 91052 Erlangen, Germany.

Received: 5 May 2015 Accepted: 16 June 2015

Published online: 09 July 2015

\section{References}

1. Headache Classification Subcommittee of the International Headache Society (2013) The International Classification of Headache Disorders, 3rd edition (beta version). Cephalalgia 33:629-808

2. Bigal ME, Serrano D, Reed M, Lipton RB (2008) Chronic migraine in the population: burden, diagnosis, and satisfaction with treatment. Neurology 71:559-566

3. Dodick DW (2006) Clinical practice. Chronic daily headache. N Engl J Med 354:158-165

4. Lipton RB, Bigal ME (2003) Chronic daily headache: is analgesic overuse a cause or a consequence? Neurology 61:154-155

5. Diener HC, Dodick DW, Aurora SK, Turkel CC, DeGryse RE, Lipton RB, Silberstein SD, Brin MF (2010) OnabotulinumtoxinA for treatment of chronic migraine: results from the double-blind, randomized, placebo-controlled phase of the PREEMPT 2 trial. Cephalalgia 30:804-814

6. Silberstein SD, Lipton RB, Dodick DW, Freitag FG, Ramadan N, Mathew N, Brandes JL, Bigal M, Saper J, Ascher S, Jordan DM, Greenberg SJ, Hulihan J (2007) Efficacy and safety of topiramate for the treatment of chronic migraine: a randomized, double-blind, placebo-controlled trial. Headache 47:170-180

7. Martelletti P, Jensen RH, Antal A, Arcioni R, Brighina F, de Tommaso M, Franzini A, Fontaine D, Heiland M, Jurgens TP, Leone M, Magis D, Paemeleire K, Palmisani S, Paulus W, May A (2013) Neuromodulation of chronic headaches: position statement from the European Headache Federation. J Headache Pain 14:86

8. Saper JR, Dodick DW, Silberstein SD, McCarville S, Sun M, Goadsby PJ (2011) Occipital nerve stimulation for the treatment of intractable chronic migraine headache: ONSTIM feasibility study. Cephalalgia 31:271-285

9. Lipton RB, Goadsby PJ, Cady RK, Aurora SK, Grosberg BM, Freitag FG, Silberstein SD, Whiten DM, Jaax KN (2009) PRISM study: occipital nerve stimulation for treatment-refractory migraine. Cephalalgia 29:30

10. Silberstein SD, Dodick DW, Saper J, Huh B, Slavin KV, Sharan A, Reed K Narouze S, Mogilner A, Goldstein J, Trentman T, Vaisman J, Ordia J, Weber P, Deer T, Levy R, Diaz RL, Washburn SN, Mekhail N (2012) Safety and efficacy of peripheral nerve stimulation of the occipital nerves for the management of chronic migraine: results from a randomized, multicenter, double-blinded, controlled study. Cephalalgia 32:1165-1179

11. Schoenen J, Vandersmissen $B$, Jeangette $S$, Herroelen $L$, Vandenheede $M$, Gerard P, Magis D (2013) Migraine prevention with a supraorbital 
transcutaneous stimulator: a randomized controlled trial. Neurology 80:697-704

12. Beekwilder JP, Beems T (2010) Overview of the clinical applications of vagus nerve stimulation. J Clin Neurophysiol 27:130-138

13. Hord ED, Evans MS, Mueed S, Adamolekun B, Naritoku DK (2003) The effect of vagus nerve stimulation on migraines. J Pain 4:530-534

14. Sadler RM, Purdy RA, Rahey S (2002) Vagal nerve stimulation aborts migraine in patient with intractable epilepsy. Cephalalgia 22:482-484

15. Mauskop A (2005) Vagus nerve stimulation relieves chronic refractory migraine and cluster headaches. Cephalalgia 25:82-86

16. Lenaerts ME, Oommen KJ, Couch JR, Skaggs V (2008) Can vagus nerve stimulation help migraine? Cephalalgia 28:392-395

17. Ellrich J (2011) Transcutaneous vagus nerve stimulation. Eur Neurol Rev 6:254-256

18. He W, Jing XH, Zhu B, Zhu XL, Li L, Bai WZ, Ben H (2013) The auriculo-vagal afferent pathway and its role in seizure suppression in rats. BMC Neurosci 14:85

19. Clancy JA, Mary DA, Witte KK, Greenwood JP, Deuchars SA, Deuchars J (2014) Non-invasive vagus nerve stimulation in healthy humans reduces sympathetic nerve activity. Brain Stimul 7:871-877

20. Kraus T, Kiess O, Hosl K, Terekhin P, Kornhuber J, Forster C (2013) CNS BOLD $\mathrm{fMRI}$ effects of sham-controlled transcutaneous electrical nerve stimulation in the left outer auditory canal - a pilot study. Brain Stimul 6:798-804

21. Frangos E, Ellrich J, Komisaruk BR (2015) Non-invasive access to the vagus nerve central projections via electrical stimulation of the external Ear: fMRI evidence in humans. Brain Stimul 8:624-636

22. Kirchner A, Birklein F, Stefan H, Handwerker HO (2000) Left vagus nerve stimulation suppresses experimentally induced pain. Neurology 55:1167-1171

23. Busch V, Zeman F, Heckel A, Menne F, Ellrich J, Eichhammer P (2013) The effect of transcutaneous vagus nerve stimulation on pain perception-an experimental study. Brain Stimul 6:202-209

24. Goadsby PJ, Grosberg BM, Mauskop A, Cady R, Simmons KA (2014) Effect of noninvasive vagus nerve stimulation on acute migraine: an open-label pilot study. Cephalalgia 34:986-993

25. Beck AT, Erbaugh J, Ward CH, Mock J, Mendelsohn M (1961) An inventory for measuring depression. Arch Gen Psychiatry 4:561-571

26. Stewart WF, Lipton RB, Dowson AJ, Sawyer J (2001) Development and testing of the Migraine Disability Assessment (MIDAS) questionnaire to assess headache-related disability. Neurology 56:S20-S28

27. Kosinski M, Bayliss MS, Bjorner JB, Ware JE Jr, Garber WH, Batenhorst A, Cady R, Dahlof CG, Dowson A, Tepper S (2003) A six-item short-form survey for measuring headache impact: the HIT-6. Qual Life Res 12:963-974

28. Freitag FG, Diamond S, Diamond M, Urban G (2008) Botulinum toxin type A in the treatment of chronic migraine without medication overuse. Headache 48:201-209

29. Silvestrini M, Bartolini M, Coccia M, Baruffaldi R, Taffi R, Provinciali L (2003) Topiramate in the treatment of chronic migraine. Cephalalgia 23:820-824

30. Diener HC, Bussone G, Van Oene JC, Lahaye M, Schwalen S, Goadsby PJ (2007) Topiramate reduces headache days in chronic migraine: a randomized double-blind, placebo-controlled study. Cephalalgia 27:814-823

31. Aurora SK, Dodick DW, Turkel CC, DeGryse RE, Silberstein SD, Lipton RB, Diener HC, Brin MF (2010) OnabotulinumtoxinA for treatment of chronic migraine: results from the double-blind, randomized, placebo-controlled phase of the PREEMPT 1 trial. Cephalalgia 30:793-803

32. The Vagus Nerve Stimulation Study Group (1995) A randomized controlled trial of chronic vagus nerve stimulation for treatment of medically intractable seizures. Neurology 45:224-230

33. Milby $\mathrm{AH}$, Halpern $\mathrm{CH}$, Baltuch $\mathrm{GH}$ (2008) Vagus nerve stimulation for epilepsy and depression. Neurotherapeutics 5:75-85

34. Ellrich J (2006) Long-term depression of orofacial somatosensory processing. Suppl Clin Neurophysiol 58:195-208

35. Ellrich J (2004) Electric low-frequency stimulation of the tongue induces long-term depression of the jaw-opening reflex in anesthetized mice. J Neurophysiol 92:3332-3337

36. Jung K, Rottmann S, Ellrich J (2009) Long-term depression of spinal nociception and pain in man: influence of varying stimulation parameters. Eur J Pain 13:161-170

37. Rottmann S, Jung K, Vohn R, Ellrich J (2010) Long-term depression of pain-related cerebral activation in healthy man: an fMRI study. Eur J Pain 14:615-624

38. Rottmann S, Jung K, Ellrich J (2010) Electrical low-frequency stimulation induces long-term depression of sensory and affective components of pain in healthy man. Eur J Pain 14:359-365
39. Jung K, Larsen LE, Rottmann S, Ellrich J (2011) Heterotopic low-frequency stimulation induces nociceptive LTD within the same central receptive field in man. Exp Brain Res 212:189-198

40. Jung K, Lelic D, Rottmann S, Drewes AM, Petrini L, Ellrich J (2012) Electrical low-frequency stimulation induces central neuroplastic changes of pain processing in man. Eur J Pain 16:509-521

41. Rottmann S, Jung K, Ellrich J (2008) Electrical low-frequency stimulation induces homotopic long-term depression of nociception and pain from hand in man. Clin Neurophysiol 119:1895-1904

42. Aymanns M, Yekta SS, Ellrich J (2009) Homotopic long-term depression of trigeminal pain and blink reflex within one side of the human face. Clin Neurophysiol 120:2093-2099

43. Yekta SS, Lamp S, Ellrich J (2006) Heterosynaptic long-term depression of craniofacial nociception: divergent effects on pain perception and blink reflex in man. Exp Brain Res 170:414-422

44. Ellrich J, Schorr A (2004) Low-frequency stimulation of trigeminal afferents induces long-term depression of human sensory processing. Brain Res 996:255-258

45. Noseda R, Burstein R (2013) Migraine pathophysiology: anatomy of the trigeminovascular pathway and associated neurological symptoms, CSD, sensitization and modulation of pain. Pain. 154 Suppl 1.

46. Peuker ET, Filler TJ (2002) The nerve supply of the human auricle. Clin Anat 15:35-37

47. Lyubashina OA, Sokolov AY, Panteleev SS (2012) Vagal afferent modulation of spinal trigeminal neuronal responses to dural electrical stimulation in rats. Neuroscience 222:29-37

48. Multon S, Schoenen J (2005) Pain control by vagus nerve stimulation: from animal to man... and back. Acta Neurol Belg 105:62-67

49. Bossut DF, Maixner W (1996) Effects of cardiac vagal afferent electrostimulation on the responses of trigeminal and trigeminothalamic neurons to noxious orofacial stimulation. Pain 65:101-109

50. Zerari-Mailly F, Buisseret P, Buisseret-Delmas C, Nosjean A (2005) Trigeminosolitarii-facial pathway in rats. J Comp Neurol 487:176-189

51. Randich A, Gebhart GF (1992) Vagal afferent modulation of nociception. Brain Res Brain Res Rev 17:77-99

52. Coppola G, Pierelli F, Schoenen J (2009) Habituation and migraine. Neurobiol Learn Mem 92:249-259

53. De Marinis M, Pujia A, Colaizzo E, Accornero N (2007) The blink reflex in "chronic migraine". Clin Neurophysiol 118:457-463

54. Aurora SK, Barrodale PM, Tipton RL, Khodavirdi A (2007) Brainstem dysfunction in chronic migraine as evidenced by neurophysiological and positron emission tomography studies. Headache 47:996-1003

55. Coppola G, lacovelli E, Bracaglia M, Serrao M, Di LC, Pierelli F (2013) Electrophysiological correlates of episodic migraine chronification: evidence for thalamic involvement. J Headache Pain 14:76

56. Henry TR (2002) Therapeutic mechanisms of vagus nerve stimulation. Neurology 59:S3-S14

57. Ben-Menachem E, Hamberger A, Hedner T, Hammond EJ, Uthman BM Slater J, Treig T, Stefan H, Ramsay RE, Wernicke JF (1995) Effects of vagus nerve stimulation on amino acids and other metabolites in the CSF of patients with partial seizures. Epilepsy Res 20:221-227

58. Richter F, Mikulik O, Ebersberger A, Schaible HG (2005) Noradrenergic agonists and antagonists influence migration of cortical spreading depression in rat-a possible mechanism of migraine prophylaxis and prevention of postischemic neuronal damage. J Cereb Blood Flow Metab 25:1225-1235

59. Pietrobon D, Striessnig J (2003) Neurobiology of migraine. Nat Rev Neurosci 4:386-398

60. Cernuda-Morollon E, Martinez-Camblor P, Alvarez R, Larrosa D, Ramon C, Pascual J (2015) Increased VIP levels in peripheral blood outside migraine attacks as a potential biomarker of cranial parasympathetic activation in chronic migraine. Cephalalgia 35:310-316

61. Schytz HW, Birk S, Wienecke T, Kruuse C, Olesen J, Ashina M (2009) PACAP38 induces migraine-like attacks in patients with migraine without aura. Brain 132:16-25

62. Bussone G, Diener HC, Pfeil J, Schwalen S (2005) Topiramate 100 mg/day in migraine prevention: a pooled analysis of double-blind randomised controlled trials. Int J Clin Pract 59:961-968 